\title{
Validação de Laboratórios Virtuais de Aprendizagem baseada em uma visão Taxonômica
}

\author{
Bárbara Gorziza Ávila ${ }^{1}$, Érico Marcelo Hoff do Amaral ${ }^{12}$, Thaísa Jacintho \\ Müller $^{134}$, Liane Margarida Rockenbach Tarouco ${ }^{1}$ \\ ${ }^{1}$ Programa de Pós-Graduação em Informática na Educação - Universidade Federal do \\ Rio Grande do Sul - Porto Alegre, RS - Brasil \\ ${ }^{2}$ Engenharia de Computação - Universidade Federal do Pampa (Unipampa) \\ Bagé, RS - Brasil \\ ${ }^{3}$ Departamento de Matemática - Pontifícia Universidade Católica do Rio Grande do Sul \\ (PUCRS) - Porto Alegre, RS - Brasil \\ ${ }^{4}$ Centro de Ciências Exatas e Tecnológicas - Universidade do Vale do Rio dos Sinos \\ (UNISINOS) - São Leopoldo, RS - Brasil \\ barbaragorzizaavila@gmail.com, erico.amaraleunipampa.edu.br, \\ thaisa.mullerepucrs.br, liane@penta.ufrgs.br
}

\begin{abstract}
Resumo. Laboratórios Virtuais de Aprendizagem (LVAs) são uma opção muito favorável para o desenvolvimento de atividades práticas sem a necessidade de utilização dos espaços da Instituição de Ensino. Entretanto, para que o seu uso seja difundido entre os docentes, é necessário que estes desenvolvam competências necessárias ao reconhecimento das ferramentas adequadas à sua prática pedagógica. Para tanto, este estudo baseia-se em uma taxonomia desenvolvida para a avaliação de LVAs no que se refere à sua estrutura e nível de interatividade. Com o intuito de validar a taxonomia, solicitou-se a um grupo de professores a avaliação de laboratórios utilizados em sua prática pedagógica, a qual demonstrou a eficácia do processo.
\end{abstract}

Palavras-chave: Laboratórios Virtuais de Aprendizagem. Taxonomia para a avaliação de LVAs. Interatividade.

\section{Validation of Virtual Learning Laboratories based on a Taxonomic vision}

Abstract. Virtual Learning Labs (LVAS) are an option very favorable for the development of practical activities without the need to use the spaces Education Institution. However, for their use is widespread among teachers, it is necessary that they develop skills needed for the recognition of the right tools to their teaching. Therefore, this study is based on a taxonomy developed for evaluating LVAS with regard to its structure and degree of interactivity. In order to validate the taxonomy, applied to a group of teachers evaluating laboratories used in their teaching practice, which demonstrated the effectiveness of the process.

Keywords: Virtual Learning Labs. Taxonomy for evaluating LVAS. Interactivity. 


\section{Introdução}

O paradigma educacional atual, com base nos preceitos de teorias como o Construtivismo e o Sócio-interacionismo, aponta para a necessidade de participação ativa do estudante no processo de ensino e aprendizagem para que seja viabilizada uma efetiva construção de conhecimento.

Para atender a demanda por atividades práticas, capazes de relacionar o conhecimento abstrato a situações concretas, professores têm buscado apoio nos Laboratórios de Aprendizagem, visto que estes se apresentam como espaços profícuos para o desenvolvimento de propostas pedagógicas que envolvem um "aprender fazendo".

Entretanto, sabe-se que as instituições de ensino dispõem de um espaço restrito no que tange aos Laboratórios de Aprendizagem, tornando-se inviável o atendimento pronto e imediato a todos os grupos de estudantes interessados no seu uso. Consequentemente, a adoção de Laboratórios Virtuais de Aprendizagem (LVAs) passa a ser uma opção válida no que se refere à possibilidade de se contemplar a todos os interessados no exercício das práticas laboratoriais.

Por outro lado, para que o uso destes recursos seja difundido entre os docentes, é necessário promover um maior conhecimento sobre suas potencialidades e limitações. $\mathrm{O}$ professor precisa não somente ter acesso às ferramentas disponíveis para o ensino de sua disciplina, mas também é necessário adquirir competências básicas que lhe permitam discernir meios eficazes para a implementação dos Laboratórios Virtuais em suas práticas pedagógicas. Neste contexto, o nível de interatividade e arquitetura oferecidos pelo LVAs são alguns dos elementos a serem observados para um bom planejamento pautado no seu uso.

Um estudo anterior, dirigido por Amaral et al (2011) desenvolveu uma taxonomia para a avaliação de Laboratórios Virtuais de Aprendizagem em função de seu nível de interatividade e arquitetura oferecida pelo sistema. Com base nesta taxonomia, este artigo conta com a avaliação de um grupo de professores sobre um conjunto de LVAs. A partir do preenchimento de um questionário, os professores apresentaram a sua visão acerca das ferramentas que costumam utilizar com seus alunos. $\mathrm{O}$ intuito desta pesquisa é promover entre os docentes uma visão crítica sobre as ferramentas que utilizam em suas atividades didáticas, além de se promover a difusão de avaliações prévias sobre ferramentas potencialmente adotáveis em todos os níveis de ensino.

A apresentação desta pesquisa ocorre em meio às seguintes seções: na seção 2 é trazido o referencial teórico, contando com a caracterização de Laboratórios Virtuais de Aprendizagem, além de serem discutidos trabalhos correlatos que deram impulso ao estudo em questão; na seção 3 apresenta-se a metodologia adotada para a avaliação dos laboratórios; a seção 4 apresenta os laboratórios e o resultado de suas avaliações pelos docentes. Por fim, a seção 5 discute as considerações finais relativas ao presente estudo.

\section{Referencial Teórico}

O reconhecimento da importância de atividades laboratoriais para a formação do indivíduo não é algo recente no âmbito educacional. Na década de 60, Kerr (1964) já argumentava sobre aspectos capazes de elevar a qualidade destes espaços para a criação de situações de ensino e aprendizagem mais eficientes e motivadoras. Segundo o autor, laboratórios de aprendizagem tendem a encorajar a observação; promover métodos científicos de pensamento; construir e aperfeiçoar habilidades de manipulação, incentivar a participação na resolução de problemas; dentre outras habilidade. 
Com base nas vantagens destacadas por Kerr, percebe-se como é válida a proposta de se explorar as práticas pedagógicas em meio a estes ambientes. Entretanto, tendo em vista que se trata de um espaço compartilhado, nem sempre é possível fazer uso deste recurso no dia a dia escolar. Devido a isso, levanta-se aqui a possibilidade de sanar as debilidades encontradas nos Laboratórios de Aprendizagem das instituições de ensino com o apoio de recursos digitais, voltados para o mesmo fim.

Neste contexto, surgem os LVAs como plataformas digitais oferecidas com o intuito de dar suporte à realização de experiências sem a necessidade da presença do usuário em um determinado local, tal como ocorre no contexto dos laboratórios reais (SCHMITT e TAROUCO, 2008). Os LVAs são aplicações baseadas somente em simulações, com representações computacionais de elementos e situações da realidade. Por não apresentar limitações com relação ao número de instrumentos ou experimentos disponíveis, deixa de existir uma obrigatoriedade de espera para se atuar em um experimento.

No âmbito da Matemática, alguns exemplos deste tipo de aplicação são o Laboratório Virtual da Unijuí e o $\mathrm{E}_{2} \mathrm{D}^{\mathrm{ii}}$, sendo ambos páginas web com exercícios interativos e propostas de investigação. Outra proposta interessante, desenvolvida recentemente no campo da Biologia, é o Ecomuve, o qual consiste em um mundo virtual desenvolvido para o ensino sobre o funcionamento de ecossistemas para estudantes do Ensino Fundamental (GROTZER et al., 2011). Cita-se ainda o Laboratório Virtual de Química, desenvolvido por Dalgarno et al. (2009) voltado para um estudo preparatório para a atuação em laboratórios presenciais.

Ao longo deste artigo, Laboratórios Virtuais de variadas áreas do conhecimento, submetidos à avaliação por docentes, serão apresentados mais detalhadamente. A seguir, descreve-se a metodologia utilizada para a seleção e avaliação dos laboratórios discutidos nesta pesquisa.

\section{Metodologia de Avaliação e Implementação}

A fim de apresentar a descrição de um método para a classificação de LVAs e por consequência sua validação, esta pesquisa foi desenvolvida com base em distintas etapas, buscando desta forma alcançar os objetivos propostos.

Em um primeiro momento partiu-se de uma revisão bibliográfica sobre LVAs, questões de interatividade e arquiteturais de tais aplicações. Esta fase caracteriza o estudo teórico sobre o tema proposto, o qual garantiu os subsídios necessários para a construção da taxonomia. Em uma segunda etapa, tem-se a proposta da taxonomia, descrita na seção 3.1, a partir de pontos pertinentes identificados no referencial teórico avaliado. Com a taxonomia fundamentalmente implementada parte-se, ainda nesta fase, para a sua avaliação, com o auxílio de um questionário (apresentado na seção 3.2), elaborado exclusivamente para este fim, caracterizando as ações práticas deste projeto. O questionário foi aplicado com professores de áreas afins aos pesquisadores, escolhidos intencionalmente por fazerem uso constante de LVAs em suas prática pedagógica. Por último, a terceira etapa destina-se à avaliação dos questionários aplicados e à apresentação e classificação, segundo a taxonomia proposta, dos LVAs descritos nas respostas destes.

\subsection{Descrição da Taxonomia}


A taxonomia proposta está calcada sobre dois padrões teoricamente reconhecidos: organização arquitetônica e nível de interatividade, ambos organizados em um gráfico de eixos X e Y, conforme a Figura 1.

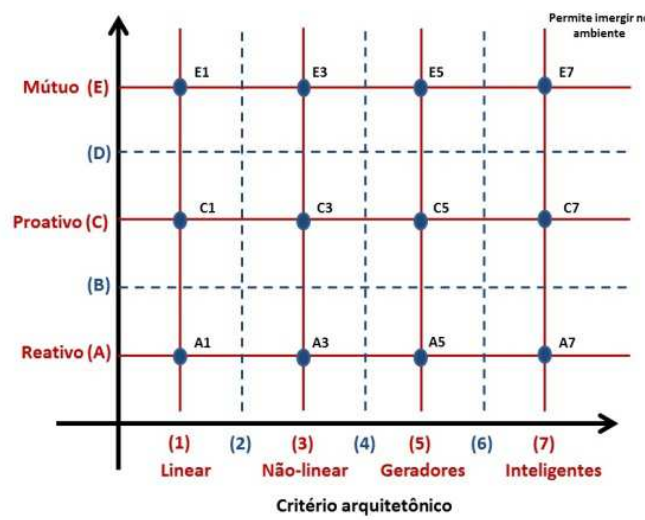

Figura 1 - Apresentação da Taxonomia

O eixo Y, vertical, apresenta o nível de interatividade proposto pelo LVA, com a pontuação descrita, em ordem crescente, desde o índice Reativo até o índice Mútuo. No eixo X, horizontal, tem-se a descrição arquitetônica do LVA, que se refere aos recursos disponibilizados pelo objeto, partindo do Linear, com recursos mínimos, até o Inteligente, apresentando soluções que permitam maior interação com a aplicação.

A utilização da taxonomia para classificação de LVAs baseia-se na observação e posterior identificação de suas características em relação à intersecção entre os eixos de Critério Arquitetônico e Nível de Interatividade (X e Y), do gráfico descrito na Figura 01. Para a correta pontuação, em relação à taxonomia, devem ser atentados os seguintes pontos:

- No que diz respeito aos Critérios Arquitetônicos, o LVA pode ser considerado Linear (1), com o funcionamento baseado no estímulo-resposta, Não-linear (2), adaptado a situações de ensino diferenciadas, em função do conhecimento do aluno e com a disponibilização de feedback, Geradores (3), quando o LVA apresenta-se organizado em forma de árvore oferecendo múltiplas oportunidades de interação, e Inteligentes (4), se o LVA apresenta recursos de inteligência artificial fornecendo uma aprendizagem colaborativa.

- Sobre os Níveis de Interatividade são utilizadas as seguintes opções para pontuar o padrão fornecido pela aplicação: Reativo (A), quando o sistema apenas responde às ações do usuário, Proativo (C), nível em que o estudante pode obter respostas diferenciadas do ambiente com uma interatividade ainda resumida, e por fim no nível Mútuo (E), no qual o sistema proporciona um alto nível de interatividade ao aprendiz.

A partir da identificação das características instrucionais do LVA, é possível utilizar a matriz proposta pela taxonomia para uma pontual identificação de qualquer laboratório. Na seção 4, serão apresentadas algumas avaliações de LVAs, seguindo estes critérios.

\subsection{Avaliação Baseada na taxonomia}

Com intuito de validar a taxonomia proposta, buscou-se um método para verificação, elencando para isso, como universo a participar da pesquisa, um conjunto de professores de Institutos Educacionais Superiores (IES), que em algum momento nas suas atividades de ensino tiveram contato com laboratórios virtuais.

Adotou-se como ferramenta um questionário contendo questões abertas e fechadas, as quais foram organizadas de modo a elencar informações gerais sobre o LVA em um primeiro momento: Indicação de data e hora; Nome do Laboratório Virtual de Aprendizagem; Público alvo; Instituição responsável pela criação; Instituição responsável pela aplicação da ferramenta; Forma de acesso ao software. Como 
informações específicas, relacionadas à validação da taxonomia, têm-se: Nome do Professor; Motivação para o uso do Laboratório; Tipo de participação oferecida pelo LVA (em termos de interação); Descrição breve sobre o contato do aluno com o sistema; Descrição sobre os níveis de colaboração disponibilizados pela aplicação; Classificação do LVA quanto à sua didática de ensino; Conteúdos explorados no LVA; breve descrição da estrutura do Laboratório Virtual de Aprendizagem; Funcionamento da estrutura frente à navegação do aluno no ambiente.

A organização destas questões vislumbra a obtenção de informações específicas para a classificação pontual em cada eixo da taxonomia, conforme mostrado na Figura 2, que apresenta uma tabela com as perguntas pertinentes ao Nível de Interatividade e Critério Arquitetônico.

\begin{tabular}{|l|l|}
\hline \multicolumn{1}{|c|}{ Nível de Interatividade } & \multicolumn{1}{c|}{ Critério Arquitetônico } \\
\hline $\begin{array}{l}\text { O Laboratório Virtual de Aprendizagem permite } \\
\text { uma participação ativa do usuário? }\end{array}$ & $\begin{array}{l}\text { A partir da sua experiência com o uso deste } \\
\text { Laboratório Virtual de Aprendizagem, qual a linha } \\
\text { de concepção pedagógica presente? }\end{array}$ \\
\hline Qual o contato do aluno com o sistema? & $\begin{array}{l}\text { O conteúdo explorado no LVA obedece alguma } \\
\text { sequência? }\end{array}$ \\
\hline $\begin{array}{l}\text { Qual o nível de colaboração disponibilizado pela } \\
\text { aplicação? }\end{array}$ & Qual o papel do aluno frente ao LVA? \\
\cline { 2 - 2 } & $\begin{array}{l}\text { Em relação à estrutura do LVA, fornece diferentes } \\
\text { caminhos, com estruturas pré-definidas ou não? }\end{array}$ \\
\hline
\end{tabular}

Figura 2 - Relação de perguntas destinadas à Classificação de LVAs segundo a taxonomia

As questões descritas na coluna da esquerda da Figura 2 estão diretamente relacionadas aos aspectos de interatividade do LVA, sendo que o resultado observado permite a pontuação da aplicação no eixo $\mathrm{Y}$ do plano cartesiano da taxonomia. Estas são perguntas de múltipla escolha, para as quais cada resposta possui um valor e o montante dos valores das questões respondidas neste item indica a faixa de classificação no Nível de Interatividade, podendo assumir um valor de 1 a 10 pontos, conforme demonstrado na Figura 3.

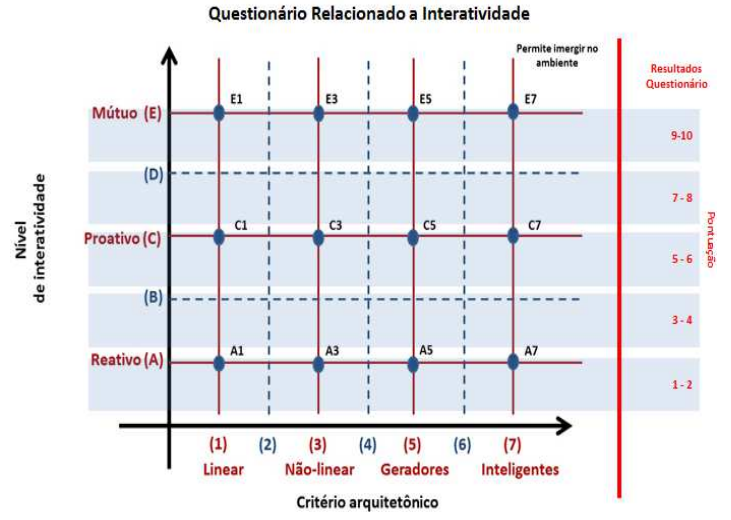

Figura 3 -Faixas de classificação no eixo Y do Plano Cartesiano da taxonomia - Nível de Interatividade

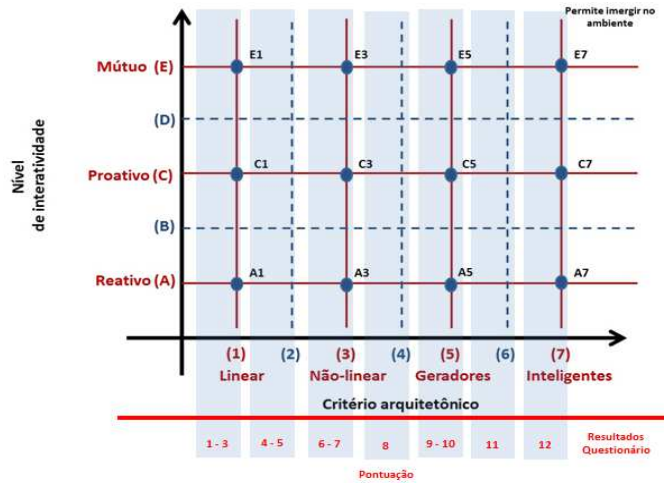

Figura 4 - Faixas de classificação no eixo X do Plano Cartesiano da taxonomia - Critério Arquitetônico

A coluna da direita da Figura 2 apresenta perguntas pontuais sobre os recursos e contato do aluno com o LVA, fornecendo desta maneira resultados que permitem identificar a quantidade de recursos disponibilizados pelo ambiente ao aluno e, desta forma, delimitar no eixo $\mathrm{X}$ do plano cartesiano da taxonomia a localização do laboratório. Assim como nas questões sobre Nível de Interatividade, cada pergunta sobre os Critérios Arquitetônicos possuem múltiplas respostas, as quais são valoradas, 
podendo alcançar ao final do questionário a uma faixa de 1 a 12 pontos, apresentados conforme a Figura 4.

A classificação final do LVA em relação à taxonomia é dada pela marcação do ponto no plano cartesiano, indicado pelo Nível de Interatividade e Critério Arquitetônico, fornecendo como resultado uma sigla que apresentará um laboratório virtual com baixo nível de interatividade e poucos recursos arquitetônicos classificados como (A1) e aplicações com alto nível de interação e com recursos de inteligência artificial com notação (E7).

\section{Resultados}

Nas seções que seguem, apresentam-se as avaliações de seis Laboratórios Virtuais de Aprendizagem, realizadas pelos professores consultados na pesquisa descrita na seção 3 . A partir das respostas encontradas para o questionário, foi feita uma análise dos laboratórios em questão. Como foi solicitado que os professores avaliassem algum laboratório que conhecessem ou já houvessem utilizado, observou-se que alguns LVAs foram avaliados por duas pessoas diferentes, e ambas as respostas serão apresentadas.

\subsection{Laboratório Didático Virtual - LabVirt}

O Laboratório Didático Virtual pertence à Faculdade de Educação da Universidade de São Paulo (USP). É constituído de simulações criadas a partir de sugestões vindas de alunos de Ensino Médio da rede pública, além de links para outros objetos de aprendizagem ou sites importantes e exemplos de projetos.

Os materiais contidos neste laboratório, que podem ser acessados livremente, são predominantemente da área de Química, de modo que seu objetivo é auxiliar alunos de licenciatura (especialmente de Química) a prepararem suas aulas, tendo como públicoalvo estes futuros professores.

O sistema é baseado na resolução de situações-problema e dá uma certa autonomia ao aluno, ainda que, após escolher o objeto a ser estudado, este siga uma sequência pré-definida. Um aspecto interessante é a possibilidade de enviar questões, as quais são respondidas por especialistas, dentro do próprio site. Este é um indício de colaboração, aspecto que quase não se encontra nas demais avaliações recebidas.

Este laboratório foi avaliado por dois professores e, considerando-se todas as respostas dadas ao questionário, foi classificado entre os níveis C3 e D6, sinalizados nos gráficos que seguem.

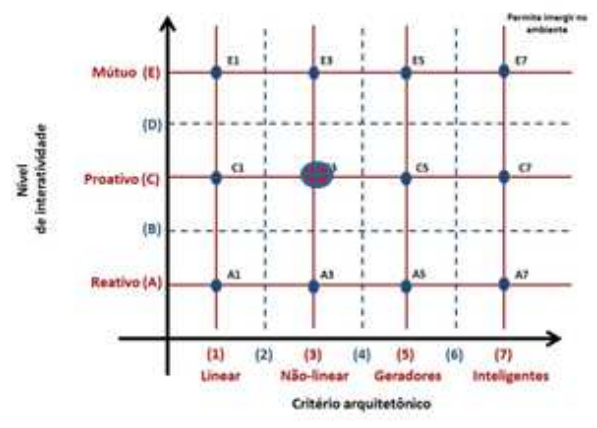

Figura 5 - LabVirt - avaliador 2

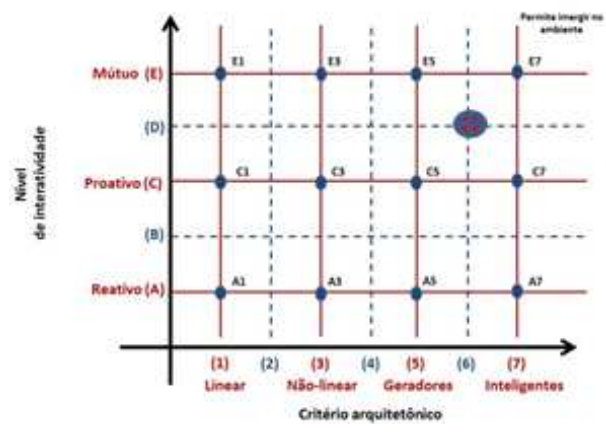

Figura 6 - LabVirt - avaliador 1

\subsection{VirtualLab}


O VirtualLab é uma aplicação composta de um conjunto de simulações na área de física, as quais contemplam diversos recursos disponíveis em um laboratório de física ou química real. Esta aplicação garante ao aprendiz atuar de forma virtual sobre um ambiente fidedigno à realidade, permitindo a tomada de decisões e ações muito próximas das previstas para um ambiente físico, observando as diferentes reações, porém garantindo a segurança durante os experimentos. O VirtualLab é uma ferramenta de acesso controlado, fornecido pela editora Pearson e que pode ser acessado por meio do sitio web < http://www.labsvirtuais.com.br/contato.asp $>$.

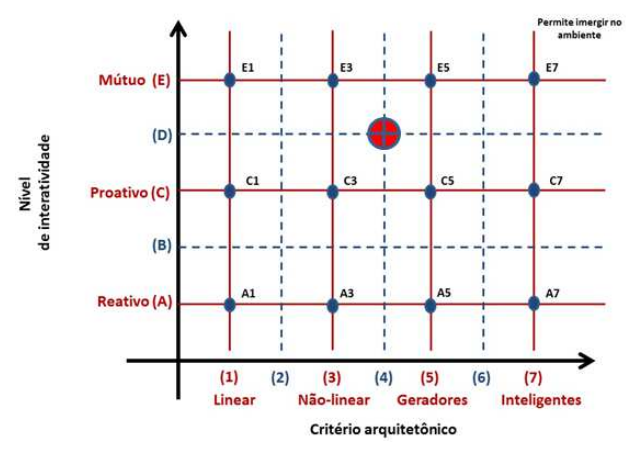

Figura 7 - Classificação do VirtualLab

porém se adequa as capacidades do aluno.

Como público alvo, tem-se professores e alunos de disciplinas de física, química e laboratórios dessas áreas, a nível de Ensino Médio ou que abordem temas como calorimetria, química quântica, gases entre outros. $\mathrm{O}$ caráter dessas aplicações e as respostas obtidas permitiram a classificação do VirtualLab como D4, conforme a Figura 7, ou seja, um nível de interação entre Proativo e Mútuo (D) com recursos entre Não-lineares e geradores, uma vez que o laboratório possui diferentes caminhos,

\subsection{Laboratório de Aprendizagem - LAPREN}

O LAPREN é um laboratório pertencente à Pontifícia Universidade Católica do Rio Grande do Sul (PUCRS), e na pesquisa aqui relatada foi avaliado por duas professoras que atuam nesta instituição. Este laboratório surgiu a partir de uma iniciativa da própria Universidade, interessada em ampliar e qualificar o serviço de monitoria oferecido a alunos de Cálculo Diferencial e Integral, disciplina conhecida pelo alto índice de reprovação nas mais diversas instituições de Ensino Superior (ROCHA, 2010). Com o crescimento do projeto, criou-se também um Laboratório Virtual, constituído por materiais criados pelos professores das Faculdades de Letras e Matemática da PUCRS.

Sendo assim, este laboratório tem como público-alvo alunos de graduação, especialmente de $1^{\circ}$ e $2^{\circ}$ níveis e, até o presente momento, seu acesso é controlado. Ao acessar o ambiente com seu login e senha, o aluno consegue navegar e selecionar entre os materiais existentes o que lhe interessa no momento. Cada um dos objetos apresenta uma parte teórica, desenvolvimento de conceitos e exercícios práticos, para que o estudante teste seu conhecimento de forma autônoma, permitindo sua participação ativa. O material possui características como a possibilidade de avanço e recuo conforme a necessidade do aluno, porém os recursos colaborativos, por exemplo, foram considerados limitados.

Com relação à classificação do LVA, observou-se, a partir das respostas das professoras consultadas, que ele foi situado, ainda que com uma pequena variação, em um nível intermediário entre proativo e mútuo, bem como entre gerador e inteligente (legendas D6 e D5). As figuras 8 e 9 abaixo mostram as duas avaliações recebidas: 


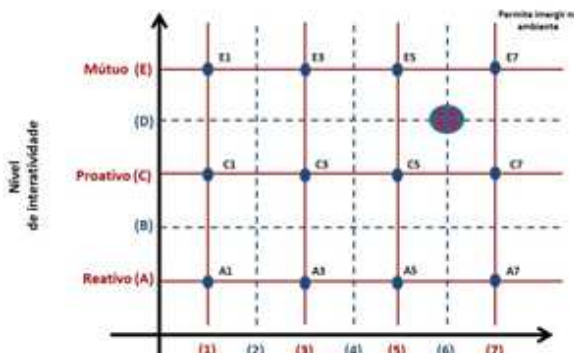

Figura 8 - LAPREN - avaliador 01

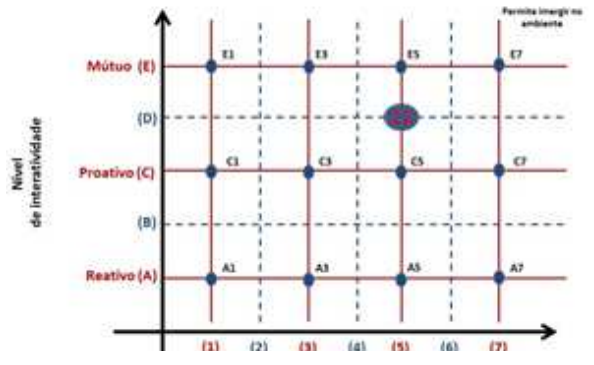

Figura 9 - LAPREN - avaliador 02

\subsection{Geogebrando (Desenvolvendo Utilitários com GeoGebra)}

Este laboratório corresponde a um projeto desenvolvido na Universidade do Vale do Rio dos Sinos (UNISINOS), em parceira com o Instituto Federal de Educação, Ciência e Tecnologia do Rio Grande do Sul (Campus Bento Gonçalves). Constitui-se em um ambiente criado inicialmente como repositório de materiais feitos com o software GeoGebra, o qual permite, através de recursos de geometria dinâmica, um alto nível de interatividade, fazendo com que o aluno participe ativamente do processo de aprendizagem. A proposta inicial era construir um local de livre acesso para compartilhamento, com professores e demais interessados, de materiais que pudessem servir de apoio às suas aulas. Com o tempo, passou a contar também com criações feitas por alunos das instituições envolvidas.

O LVA é constituído por diversas partes, objetivando o aprendizado de alguns conteúdos de Matemática (tanto de nível básico como superior), de modo que é possível navegar e explorar as seções conforme a conveniência. Através de experimentos de Geometria, Funções e etc., pode-se afirmar que o aluno é desafiado a pensar e resolver problemas de forma autônoma. Da mesma forma que no laboratório anterior, existe a limitação de não apresentar ferramentas colaborativas, de modo que a própria inclusão de materiais criados por estudantes, comentada acima, deve necessariamente passar pelos moderadores.

Com base nestas observações e nas respostas obtidas no questionário pelo professor que escolheu este LVA para participar da pesquisa, apresenta-se na Figura 10 sua classificação no nível D5.

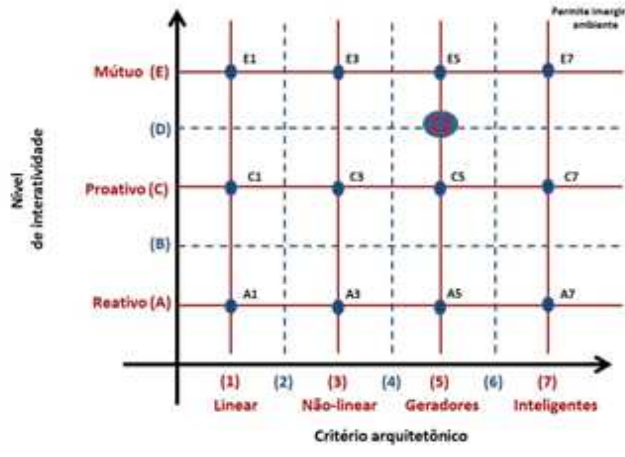

Figura 10 - Geogebrando

\subsection{Packet Tracer}

O Packet Tracer (PT) é um software desenvolvido pela Empresa Cisco, líder mundial em equipamentos e componentes para infraestutra de redes, disponível em http://www.cisco.com/web/learning/netacad/course_catalog/PacketTracer.htm. O intuito desta ferramenta é apoiar na formação de profissionais da área de redes de computadores, por meio de um conjunto de simulações das atividades mais comuns na configuração de um ambiente de redes. O PT destaca-se por possibilitar ao aluno identificar o comportamento lógico e físico de diferentes componentes em um ambiente de rede simples ou com alto grau de complexidade, sem a necessidade de aquisição de equipamentos reais para este fim. 
Este LVA tem como público alvo profissionais e estudantes da área de redes de computadores, que busquem se qualificar sobre temas tecnológicos nesta área, por meio da utilização de um sistema que permita a simulação ativa de conceitos e práticas técnicas em um projeto de redes, visando aprimorar a capacidade de tais alunos na tomada de decisão e resolução de problemas.

Sobre as respostas relacionadas a este LVA, foi possível identificar que as descrições dos participantes convergiram a uma mesma pontuação, a qual caracterizou o PT como D5 pelo primeiro avaliador e D6 pelo segundo. A diferença apresentada de um ponto no critério arquitetônico não desconfigura a proximidade das interpretações de tais avaliadores, assim como não muda de forma drástica a interpretação da classificação. Em ambas a interação demonstrou-se acima do nível interativo com um critério arquitetônico pontualmente Gerador na primeira avaliação e acima de Gerador pelo segundo professor, conforme as Figuras 11 e 12.

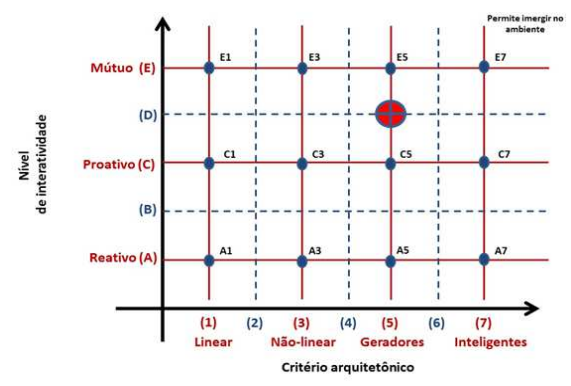

Figura 11 - PT - avaliador 01

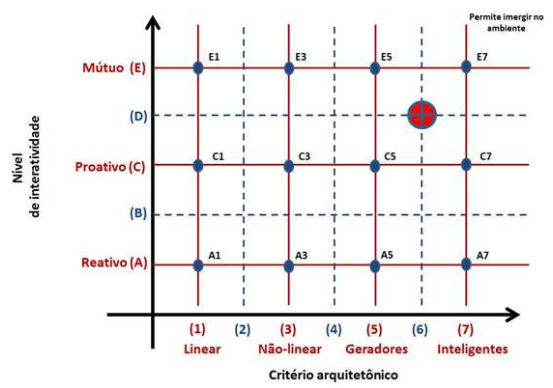

Figura 12 - PT - avaliador 02

\subsection{Ensino de Derivadas a Distância - E2D}

$\mathrm{O} \mathrm{E}_{2} \mathrm{D}$ é uma ferramenta criada por profissionais das áreas de Matemática e de Informática, alunos do programa de Pós Graduação em Informática na Educação da Universidade Federal do Rio Grande do Sul. Surgiu como um objeto de aprendizagem voltado a apoiar o ensino de Cálculo, mais especificamente do conteúdo derivadas, tendo como público alvo alunos que cursam esta disciplina.

Porém, o $\mathrm{E}_{2} \mathrm{D}$ pode também ser considerado como um LVA e avaliado como tal, uma vez que permite de forma muito evidente a experimentação, através de recursos interativos, levando o aluno a construir o conceito de derivadas através da manipulação de gráficos e retas tangentes, por exemplo, o que é possível mais uma vez através do uso do software GeoGebra.

A implementação deste projeto e suas possibilidades já foi discutida por seus autores (AMARAL e MÜLLER, 2012), e o ambiente, que possui acesso livre, avaliado por professores da área de Matemática. Com o olhar mais voltado ao uso do ambiente como um LVA, a professora que utilizou o $\mathrm{E}_{2} \mathrm{D}$ para responder ao questionário solicitado na pesquisa aqui apresentada classificou-o entre os níveis proativo e mútuo, bem como geradores e inteligentes, situando-o no ponto D5. Isto porque, como se pode observar na respostas obtidas permite várias aluno com o aprendizagem apresenta

V. $10 \mathrm{~N}^{\mathrm{o}} 3$, dezembro, 2

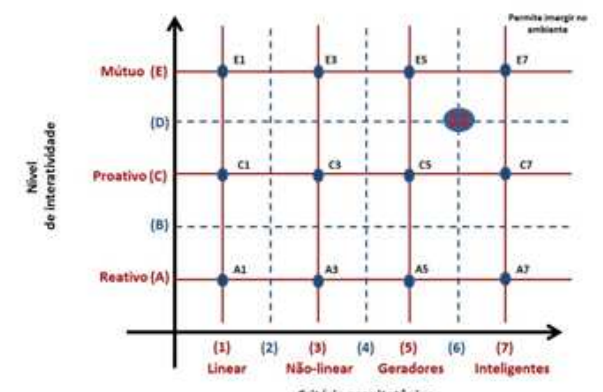
ferramenta e também nas na pesquisa, o ambiente formas de interação do sistema, enfatiza a ativa, mas também limitações na questão 
colaborativa. Outras questões e observações contidas na avaliação deste LVA foram resumidas para a obtenção do resultado final, apresentado na Figura 13.

Figura 13 - Ensino de Derivadas a Distância

\section{Considerações Finais}

Neste estudo foi realizado um mapeamento de LVAs existentes na rede, em sua maioria de acesso livre, o que certamente será bastante útil para auxiliar professores em sua busca por materiais capazes de promover maior qualidade ao ensino.

De um modo geral, os participantes da pesquisa eram professores, e os laboratórios avaliados têm como público alvo estudantes dos mais variados níveis de ensino. Além disso, tais laboratórios referem-se às ciências exatas, servindo de apoio ao ensino de conteúdos de química, física, matemática e informática. Este fato está diretamente relacionado à formação dos autores, que os levou a contatos com profissionais de áreas afins.

Com base nos dados apresentados, conforme Amaral et al (2011), observa-se que todos os laboratórios avaliados enquadraram-se nos tipos proativo ou mútuo (quanto à interatividade) e não-lineares, geradores ou inteligentes (quanto ao critério arquitetônico).

Nenhum dos laboratórios foi classificado no nível de reativo ou linear, ou seja, todos oferecem ao menos um nível intermediário de arquitetura capaz de promover maior interatividade, tornando mais envolvente a participação do aluno nos experimentos propostos.

Outro fato interessante é que os laboratórios que foram avaliados por duas pessoas diferentes não apresentaram exatamente o mesmo resultado, mas as avaliações foram bem semelhantes, o que auxilia na confirmação das respostas e aponta também para a eficiência da matriz de classificação adotada.

\section{Referências}

AMARAL, Erico; AVILA, Barbara; ZEDNIK, Herik; TAROUCO, Liane. Laboratório Virtual de Aprendizagem: Uma Proposta Taxonômica. Renote. v. 9 n. 2, p. 1-10, 2011.

AMARAL, Erico; MULLER, Thaísa. Integração de Tecnologias para Construção de Objetos de Aprendizagem - $\mathrm{O}$ case $\mathrm{E}_{2} \mathrm{D}$ Ensino de Derivadas a Distância. Renote. v. 10 n. 1, p. 1-11, 2012.

DALGARNO, Barney; BISHOP, Andrea G.; ADLONG, Willian; BEDGOOD JR., Danny R. Effectiveness of a Virtual Laboratory as a preparatory resource for Distance Education chemistry students.Computers \& Education, v. 53, p. 853-865, Mai/2009. 
GROTZER, Tina; TUTWILER, Shane; DEDE, Chris; KAMARAINEN, Amy; MATCALF, Shari. Helping Students Learn More Expert Framing of Complex Causal Dynamics in Ecosystems Using EcoMUVE. In: Science Teaching (NARST) Conference. Orlando, FL, April 4, 2011. p. 1-15.

KERR, John F. Pratical work in school science: an account of an inquiry into the nature and purpose of pratical work in school science in England an Wales. Leicester: Leicester University press, 1964.

ROCHA, M. D. Desenvolvimento de atividades computacionais na disciplina de Cálculo Diferencial e Integral I: estudo de uma proposta de ensino pautada na articulação entre a visualização e a experimentação. Ouro Preto: Programa de PósGraduação em Educação Matemática, 2010. 172 p. Dissertação de mestrado. Universidade Federal de Ouro Preto, 2010.

SCHMITT, M. A. R.; TAROUCO, L. M. R. Metaversos e laboratórios virtuais possibilidades e dificuldades. Revista de Novas Tecnologias na Educação, Porto Alegre, v. 6, n. 1, p 1-12, jul/2008.

\footnotetext{
${ }^{\mathrm{i}}$ http://www.projetos.unijui.edu.br/matematica/

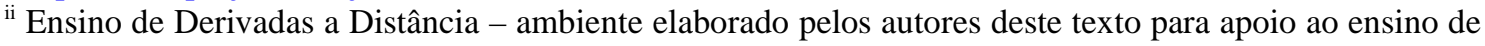
Cálculo. Disponível em http://www.scutum.com.br/e2d/index.html.
} 